\title{
Correlative Bioimaging with Electron, X-ray and Light Microscopies
}

\author{
M.A. Aronova ${ }^{1}$, S. Chen ${ }^{2}$, B. Lai ${ }^{2}$, N.B. Pivovarova ${ }^{3}$, A. Daoust ${ }^{3}$, S.B. Andrews ${ }^{3}$, A.P. Koretsky ${ }^{3}$, R.D. \\ Leapman $^{1}$ \\ 1. National Institute of Biomedical Imaging and Bioengineering, NIH, Bethesda, MD, USA \\ 2. Advanced Photon Source, Argonne National Laboratory, Argonne, IL, USA \\ 3. National Institute of Neurological Disorders and Stroke, NIH, Bethesda, MD, USA
}

Correlative microscopy has become an essential technique for determining the relationship between structure and function in cells and tissues on the scale of subcellular organelles and supramolecular assemblies [1]. We have used this approach to combine the nanoscale spatial resolution of electron microscopy (EM) with optical fluorescence imaging to localize specific labeled proteins. Here, we also correlate EM with X-ray fluorescence imaging to localize specific types of diffusible ions.

We first review previous work on correlating energy-filtered transmission EM (EFTEM) images with optical images of specific cellular proteins in plastic sections of cells labeled using antibodies conjugated to fluorescent tags. The resulting elemental distributions obtained in the EFTEM can distinguish between biological molecules, such as nucleic acids containing phosphorus and nitrogen, and proteins containing nitrogen but little phosphorus [2]. In cryo-EM of frozen hydrated samples, it is possible to correlate optical images of genetically encoded fluorophores, e.g., green fluorescent proteins, directly with high-resolution phase-contrast TEM images [3]. Furthermore, by using antibodies doublelabeled with both easily visible fluorescent tags and nanometer-diameter gold atom clusters, which can be imaged by dark-field scanning transmission electron microscopy (STEM), it is possible to localize labeled proteins in the EFTEM images with high precision [4]. Recently, correlative techniques have been further extended to include multicolor EFTEM probes containing rare earth elements that facilitate the labeling of multiple specific cellular proteins using genetically encoded, photoactivated, singlet oxygen generators [5]. On activation with light of different wavelengths, these labels polymerize different rare-earth chelates of diaminobenzidine, which are introduced successively into the specimen; and after sample preparation, the rare-earth atoms are retained near to the locations of the original genetic tags and can be imaged by EFTEM.

Secondly, in the present study, we use correlative imaging to elucidate the mechanism for uptake of manganese ions in $\mathrm{Mn}^{2+}$ enhanced magnetic resonance imaging of brain, which enables the tracing of neuronal connections [6]. Specimens were prepared by slam-freezing organotypic rat hippocampal slices (cultured in $25 \mu \mathrm{M} \mathrm{Mn}^{2+}$ for $24 \mathrm{~h}$ ). Cryosections cut to a nominal thickness of $80 \mathrm{~nm}$ were mounted on copper EM grids and cryo-transferred at liquid nitrogen temperature to an FEI TF30 TEM (Thermo Fisher Scientific). The cryosections were freeze-dried at $-90{ }^{\circ} \mathrm{C}$ for 20 minutes and were imaged at a dose of $<10^{4}$ electrons $/ \mathrm{nm}^{2}$. Grids were cryo-transferred back from the microscope and shipped cold to Argonne National Laboratory. X-ray elemental images were acquired using the X-ray Bionanoprobe at the Advanced Photon Source [7], and $\mathrm{Mn}^{2+}$ distributions were correlated with TEM ultrastructure.

Fig. 1 shows correlative TEM and X-ray nanoprobe imaging of manganese in two CA1 hippocampal neurons, from which it is evident that the $\mathrm{Mn}^{2+}$ is concentrated in Golgi, consistent with a previously reported detoxification process in which $\mathrm{Mn}^{2+}$ is sequestered into Golgi vesicles [8]. This result illustrates the value of correlative imaging to localize low concentrations of specific ions in organelles 
that are structurally well characterized [9].

References:

[1] I Begemann and M Galic, Frontier Synaptic Neurosci. 8 (2016) article 28, p. 1.

[2] Y Ren, MJ Kruhlak and DP Bazett-Jones, J. Histochem. Cytochem. 51 (2003) p. 605.

[3] A Sartori et al, J. Struct. Biol. 160 (2007), p. 135.

[4] MA Aronova et al, Microsc. Microanal. 15 (Suppl. 2) (2009), p. 920.

[5] SR Adams et al, Cell Chem. Biol. 23 (2016), p. 1417.

[6] A Daoust et al, Proc. 26 ${ }^{\text {th }}$ ISMRM Annual Meeting (2018), in press.

[7] S Chen et al, J. Synchrotron Rad. 21 (2014), p. 66.

[8] A Carmona et al, ACS Chem. Neurosci. 1 (2010), p. 194.

[9] Research supported by the intramural programs of NIBIB and NINDS at the National Institutes of Health. This research used resources of the Advanced Photon Source, a U.S. Department of Energy (DOE) Office of Science User Facility operated for the DOE Office of Science by Argonne National Laboratory under Contract No. DE-AC02-06CH11357
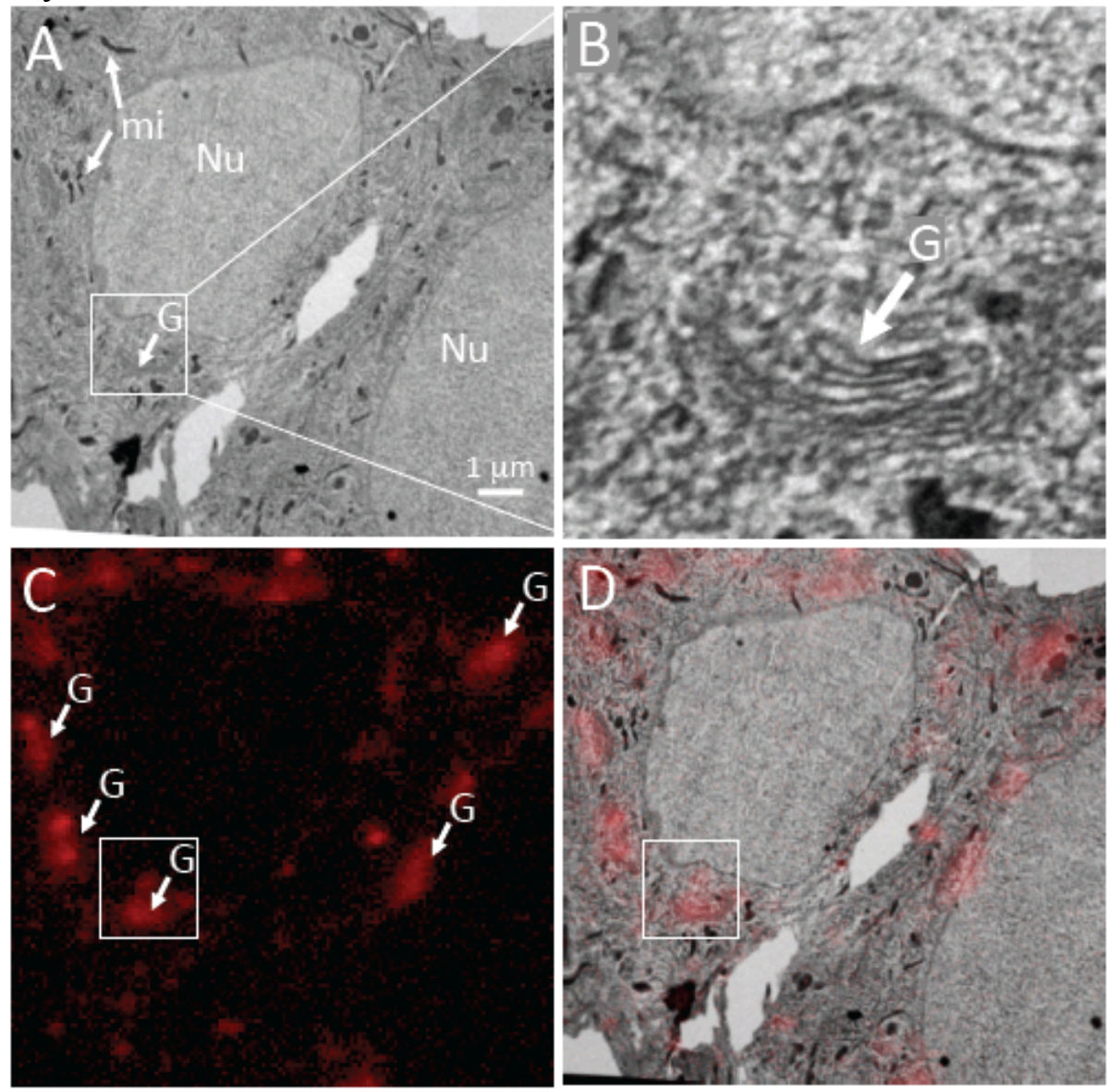

Figure 1. Correlative TEM and X-ray Bionanoprobe imaging of CA1 region neurons in freeze-dried, cryosection of rapidly frozen rat hippocampal slice culture. (A) TEM showing nuclei $(\mathrm{Nu})$, mitochondria (mi) indicated by arrows, and Golgi $(\mathrm{G})$ indicated by arrow in boxed region. (B) Higher magnification of boxed region revealing Golgi membranes (G). (C) X-ray image of $\mathrm{Mn}^{2+}$ (red), showing accumulation in Golgi $(\mathrm{G})$ indicated by arrows but absence in mitochondria and nuclei. The highest local concentrations of $\mathrm{Mn}^{2+}$ are $\sim 15 \mathrm{mmol} / \mathrm{l}$. (D) Overlay of TEM and manganese X-ray map reveals strong correlation of $\mathrm{Mn}^{2+}$ with perinuclear Golgi organelles. 